\title{
Image orientation for three-dimensional echocardiography of congenital heart disease
}

\author{
John Simpson • Owen Miller • Aaron Bell • \\ Hannah Bellsham-Revell • Jackie McGhie • \\ Folkert Meijboom
}

Received: 12 November 2010/Accepted: 19 May 2011/Published online: 31 May 2011

(C) Springer Science+Business Media, B.V. 2011

\begin{abstract}
To date there has been little discussion about image orientation for three-dimensional (3D) echocardiography when applied to congenital heart lesions. Anatomic relations cannot be assumed in congenital heart disease and image cropping during post processing may by necessity remove external or even internal anatomic references. We present an approach to consistent anatomic orientation which is both intuitive and consistent with regard to superiorinferior, anterior-posterior and left-right axes. Such anatomic orientation is also concordant with other common 3D imaging modes such as cardiac magnetic resonance imaging and computed tomography. Views derived from standard cross sectional echocardiography have such universal familiarity that analogous 3D projections of these views may be retained but novel hitherto unavailable views such as en face views of
\end{abstract}

J. Simpson $(\varangle) \cdot$ O. Miller · A. Bell ·

H. Bellsham-Revell

Department of Congenital Heart Disease, Evelina Children's Hospital, Guy's and St Thomas' NHS

Foundation Trust, London SE1 7EH, UK

e-mail: john.simpson@gstt.nhs.uk

J. McGhie

Department of Cardiology, Thoraxcenter, Erasmus

Medical Center, Rotterdam, The Netherlands

F. Meijboom

Departments of Cardiology and Pediatrics, University

Medical Centre Utrecht, Utrecht, The Netherlands the cardiac septums or atrioventricular valves may be projected using anatomic orientation.

Keywords Three dimensional echocardiography . Congenital heart disease - Image orientation

\section{Introduction}

Three-dimensional (3D) echocardiography has become an integral part of the echocardiographic interrogation of the heart in recent years. The two major areas where 3D echocardiography is clinically applied are the assessment of cardiac function and imaging of cardiac anatomy. 3D echocardiography is being increasingly employed for the evaluation of a wide range of congenital heart defects both for surgical planning or to guide interventions $[1,2]$. The indications for the technique and its current limitations with respect to congenital heart disease have been reviewed recently [3]. In the $3 \mathrm{D}$ echocardiographic assessment of left ventricular function, there is a relatively high degree of consistency in the presentation using the 17 segment model of the left ventricle [4]. This contrasts with the presentation of structural information which is far more variable.

In 2D echocardiography, cut planes have been largely determined by accessible acoustic windows. The presentation of $2 \mathrm{D}$ views is relatively standardized although there may be some variation mainly 
relating to whether the cardiac apex is displayed up or down in the apical 4 chamber view or subcostal views [5]. In its simplest form, 3D echocardiography using standard 2D image planes can simply add depth to an image which is already familiar. However, the real strength of the $3 \mathrm{D}$ echocardiographic technique is the generation of a volume dataset which can be post-processed and analysed using unrestricted cropping planes and spatially orientated by rotation around Cartesian axes [6]. There are a number of different 3D acquisition modalities including full-volume, live and "zoom" modalities which differ with respect to the field of view, spatial and temporal resolution [7]. Full-volume acquisitions have a wide field of view and are typically acquired over several cardiac cycles, which can introduce a problem of artefact related to patient movement. Live 3D echocardiography has a narrower field of view but avoids the problem of patient movement. Zoom modalities concentrate rendering of $3 \mathrm{D}$ images on a user-defined region but has a limitation of low temporal resolution if a large area of interest is selected. Details of these modalities are reviewed elsewhere [3, 7]. Whatever the modality of acquisition, cropping of a 3D volume dataset can produce hitherto unfamiliar and novel views, for example en face images of structures such as the atrial septum, the ventricular septum and the atrioventricular valves. Even for experienced echocardiographers, the interpretation of these novel 3D projections, not previously seen with the $2 \mathrm{D}$ technique, will involve an initial learning curve. Understanding such novel views may be even more challenging for clinicians, such as surgeons and interventional cardiologists who may not perform echocardiography but nonetheless need to understand these images to guide intervention and surgery.

It is these new and potentially unfamiliar views which may benefit most from a consistent anatomical approach by orienting the view to consistent superior-inferior and left-right axes and to identifiable structural landmarks. This is particularly relevant in the congenitally abnormal heart where, in contrast to the normally connected heart, the relationship of one cardiac structure to another cannot be assumed. Other volumetric imaging modalities, such as magnetic resonance imaging (MRI) and computed tomography (CT) have already closely followed an anatomic model in display orientation [8].
We describe an approach to image orientation of standard and novel 3D echocardiographic views, which is both intuitive and consistent with other imaging techniques. The approach described does not attempt to evaluate the relative diagnostic merits of cross-sectional echocardiography versus 3D echocardiography or MRI but solely addresses the question of image orientation.

\section{Methods}

Principles of approach

The principles of the approach which have been adopted can be summarised as follows:

1. Image projections analogous to standard 2D echocardiographic planes, can be retained, but in some cases may benefit from minor adjustment to an anatomic axis.

2. 3D echocardiographic images of novel projections such as en face views of cardiac septums and the "surgical view" of the atrioventricular junction should be presented in a consistent orientation with intuitive left/right, superior/inferior orientation.

Comparison of orientation technique with other modalities

Other volumetric imaging methods, such as MRI and $\mathrm{CT}$, maintain external anatomic reference structures and also employ an orientation key or tool which rotates and angulates as the dataset is manipulated maintaining a constant relationship between Cartesian $\mathrm{x}, \mathrm{y}, \mathrm{z}$ axis planes and the anatomic orientation. This is not the case for 3D echocardiography, where a reference axis tool is lacking and external anatomic relations and internal cardiac references may be lost when images are cropped during post-processing.

\section{Standard views}

The apical four chamber view

Although the apical four chamber view is well known to all using standard 2D echocardiography, due to depth of field in 3D echocardiography, additional 
structures can now be displayed, such as the inferior vena cava draining to the floor of the right atrium (Fig. 1). Institutional practice and the preference of the echocardiographer will dictate whether the $3 \mathrm{D}$ image is displayed apex up or apex down.

\section{Parasternal long axis view of the left ventricle}

The standard long axis view developed historically as it is readily obtained from a parasternal view and presents the ventricular septum optimally to facilitate M-mode analysis [9]. 3D echocardiography from a standard parasternal long axis approach can add depth/width to normal structures such as the mitral valve leaflets but also abnormalities for example, a subaortic membrane (Fig. 2).

\section{Parasternal short axis views}

The standard 2D parasternal short axis approach can be employed for 3D echocardiographic imaging. At the mid septal level such a projection may include far field structures allowing both mitral and aortic valves to be seen in the same image. This can also add detail

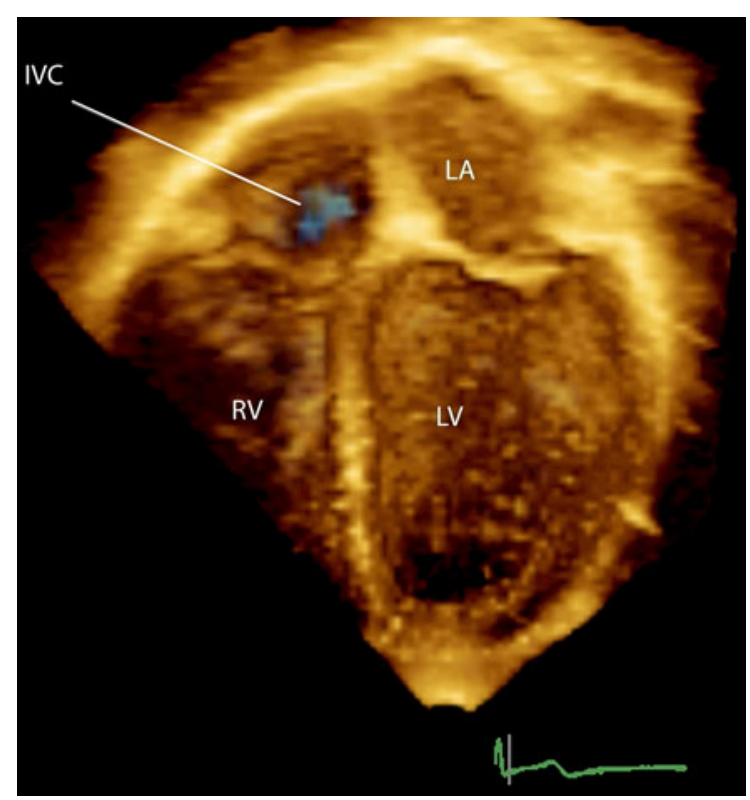

Fig. 1 Four chamber view of the heart. A projection of the four chamber view with the apex down. The inferior vena cava is seen draining to the right atrium. The great arteries are out of plane anteriorly. $L V$ left ventricle, $R V$ right ventricle, $L A$ left atrium, $I V C$ inferior vena cava

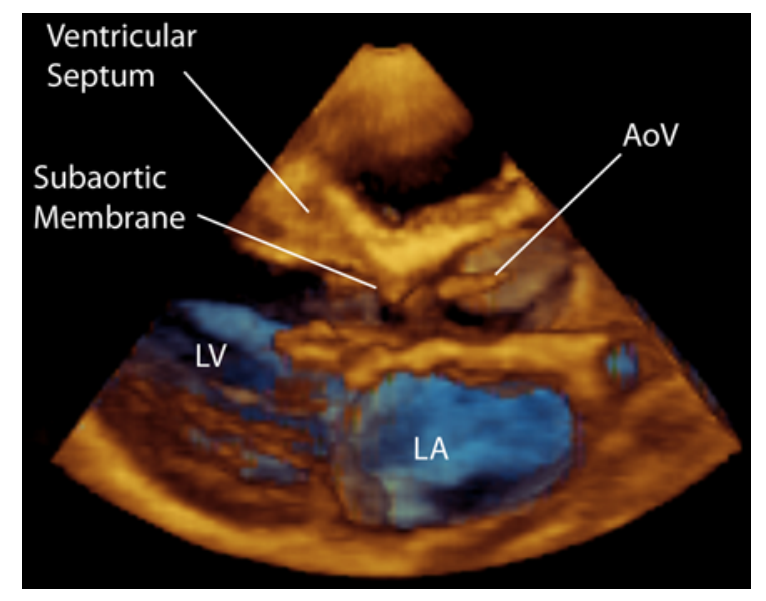

Fig. 2 3D image adding depth to a standard long axis view of the left ventricle with enhanced definition of a subaortic membrane. $A o V$ aortic valve, $L A$ left atrium, $L V$ left ventricle

and depth when displaying a ventricular septal defect (Fig. 3).

\section{External landmarks}

Although standard 2D planes can be used for 3D echocardiography, they are defined by the point and orientation of insonation and the orientation of the image cannot be altered following acquisition. 3D

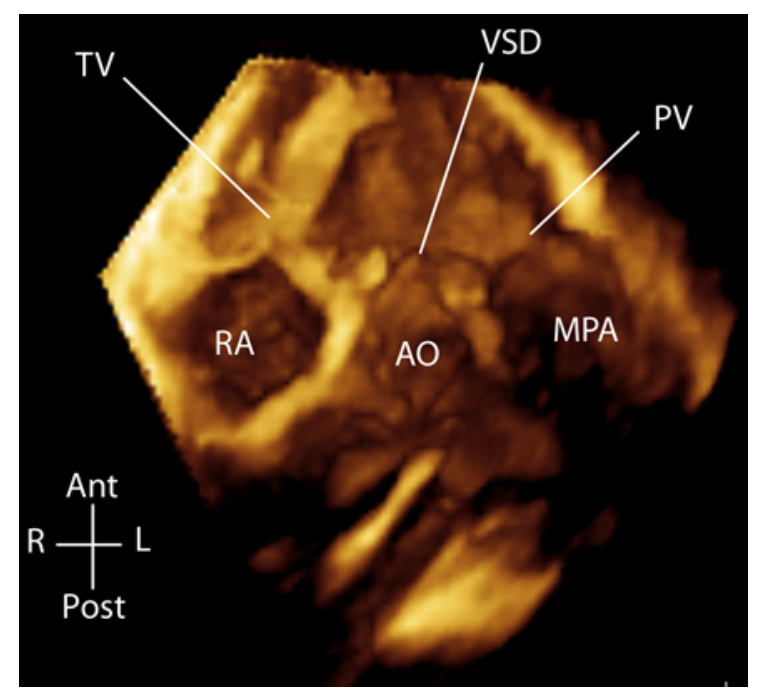

Fig. 3 3D parasternal short axis view at the level of the aortic valve profiles the margins of the ventricular septal defect. $T V$ tricuspid valve, $V S D$ ventricular septal defect, $P V$ pulmonary valve, $M P A$ main pulmonary artery, $A o$ aorta, $R A$ right atrium, $L A$ left atrium, Ant anterior, Post posterior, $L$ left, $R$ right 
echocardiographic image projection, however, can be altered during post processing and the anatomic relations can be adjusted with reference to known landmarks such as the diaphragm. This may be particularly useful when displaying 3D echocardiography alongside other imaging modalities.

\section{Unique 3D echocardiographic views}

\section{Views of normal atrioventricular valves}

One of the recognised strengths of 3D echocardiographic imaging is that en face views of the atrioventricular valves can be produced. The atrioventricular valves can therefore be presented from either the ventricular aspect or from the atrial aspect of the valve as if viewed by an observer who is directly facing the valve in question.

\section{The mitral valve}

The mitral valve is frequently projected in short axis as if viewed from the cavity of the left ventricle. True anatomic orientation of this projection is confirmed with reference to MRI (Fig. 4a) where the relationship of the papillary muscles is key. 3D echocardiographic images of the short axis of the left ventricle, papillary muscles and mitral valve, can be orientated to match this anatomic projection (Fig. $4 b, c)$. The resulting images maintain the inferior wall of the left ventricle lowermost in the projected image. The echocardiographic technique has the major advantage of showing valve leaflets and the left ventricular outflow tract clearly in 3D rendered views. If this anatomically orientated short axis of the left ventricle is viewed as a clock-face, then the left ventricular outflow tract is seen at around 10 o'clock (Fig. 4c) when viewed from the left ventricle.

\section{The tricuspid valve}

The same principles that were applied to imaging of the mitral valve can be applied to the tricuspid valve, resulting in anatomic orientation of the valve, whether viewed from the ventricular or the atrial aspect of the valve. The en face plane for the tricuspid valve is also demonstrated by the MRI orientation in Fig. 4a. 3D echocardiography of the tricuspid valve is shown when viewed either from the ventricle (Fig. 5a) or from the right atrium (Fig. 5b).

Views of an abnormal atrioventricular or ventriculoarterial junction

The principles of an anatomic approach to 3D image orientation are particularly pertinent in the context of congenital abnormalities. In the atrioventricular septal defect, true anatomical orientation with reference to the diaphragm allows the valve morphology to be displayed with the superior bridging leaflet shown uppermost and the inferior bridging leaflet lowermost (Fig. 6). Use of the plane of the diaphragm to mark the inferior border of the heart may be similarly applied to subcostal, short axis and novel en face projections.

In ventriculo-arterial discordance, particularly with a ventricular septal defect (VSD), surgical options include baffling the left ventricular outflow via the VSD to the aortic root which arises from the right heart. Figure 7 shows the relationship between the mitral valve, the VSD and the anterior and rightward aorta.

\section{"Surgical" views of the atrioventricular valves}

To date transthoracic and epicardial 3D echocardiography has provided enhanced imaging beyond conventional two dimensional echocardiography. The recent introduction of transoesophageal 3D echocardiography has facilitated a further evolution in imaging the atrioventricular valves. Most importantly, novel views of the valve surface as if viewed from the atrial chamber, are analogous to the actual views the surgeon will see at the time of operation. Employing consistent anatomical orientation (Fig. 8), the atrioventricular junction has the aorta shown superiorly on the image, the mitral valve to the left and the tricuspid valve to the right. The diaphragmatic surface of the heart is lowermost.

\section{Aortic valve}

$3 \mathrm{D}$ echo of the aortic valve has become increasingly important to assess the aortic valve morphology particularly in cases of aortic stenosis and regurgitation. The 3D en face view can provide information on the mechanism of closure and location of regurgitant jets. The shape and surface area of the effective orifice can also be evaluated. 3D echocardiography may be used for 

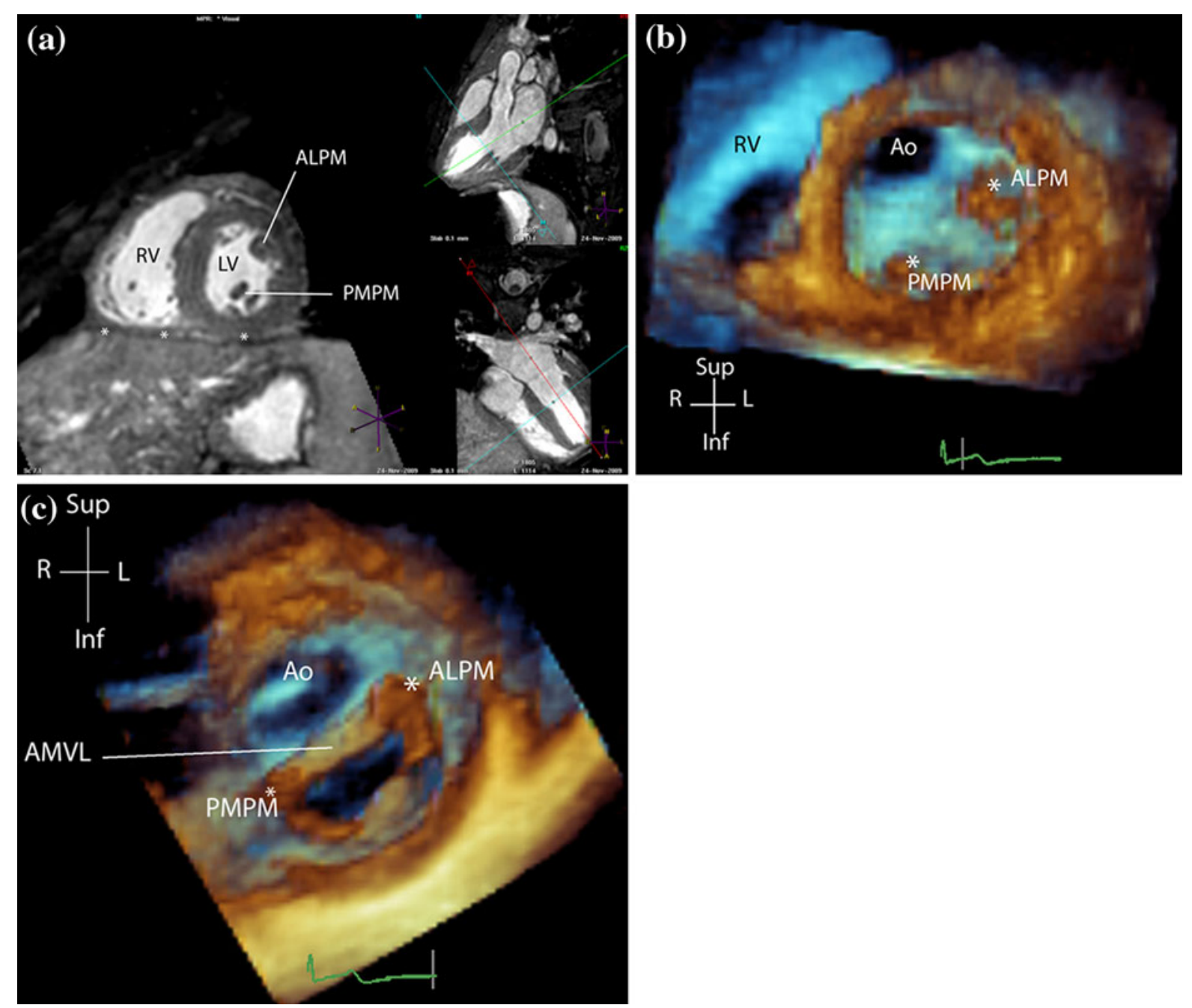

Fig. 4 a MRI "short axis" slice with en face projection from the left ventricular apex towards the papillary muscles. Preservation of external anatomic landmarks is a strength of MRI imaging. The horizontal diaphragmatic surface shown by the asterisks demonstrates the correct orientation of the papillary muscles. $R V$ right ventricle, $L V$ left ventricle, $A L P M$ anterolateral papillary muscle, $P M P M$ posteromedial papillary muscle, Ant anterior, Post posterior, $L$ left, $R$ right, $H$ head, $F$ foot. b 3D echocardiographic image of left ventricle and papillary muscles rotated and displayed similarly to MRI in

the selection of patients who can be candidates for aortic valve repair (instead of replacement) and for guiding interventions, both surgical and catheter-based.

When the aortic valve is imaged en face by transthoracic 3D echocardiography, as if looking from the ventricle towards the aortic valve, orientation is analogous to the cross-sectional orientation (Fig. 9). However, when transoesophageal
Fig. 4a. The papillary muscles are marked by asterisks and labelled. 3D depth allows the aorta to be visualised in the far field. $R V$ right ventricle, $A o$ aorta, $A L P M$ anterolateral papillary muscle, $P M P M$ posteromedial papillary muscle, Sup superior, Inf inferior, $L$ left, $R$ right. c Left ventricle, mitral valve and papillary muscles orientated anatomically. Ao aorta, ALPM anterolateral papillary muscle, $P M P M$ posteromedial papillary muscle, $A M V L$ anterior mitral valve leaflet, Sup superior, Inf inferior, $L$ left, $R$ right

echocardiography (TOE) is used to image the aortic valve, due to the retrocardiac position of the TOE probe, the short axis orientation is reversed in the anterior/posterior axis (Fig. 10). Although this TOE view is not "anatomic" as the more anterior structures appear lowermost on the image, there is a high degree of familiarity with the standard 2D TOE view. For these reasons many echocardiographers may 

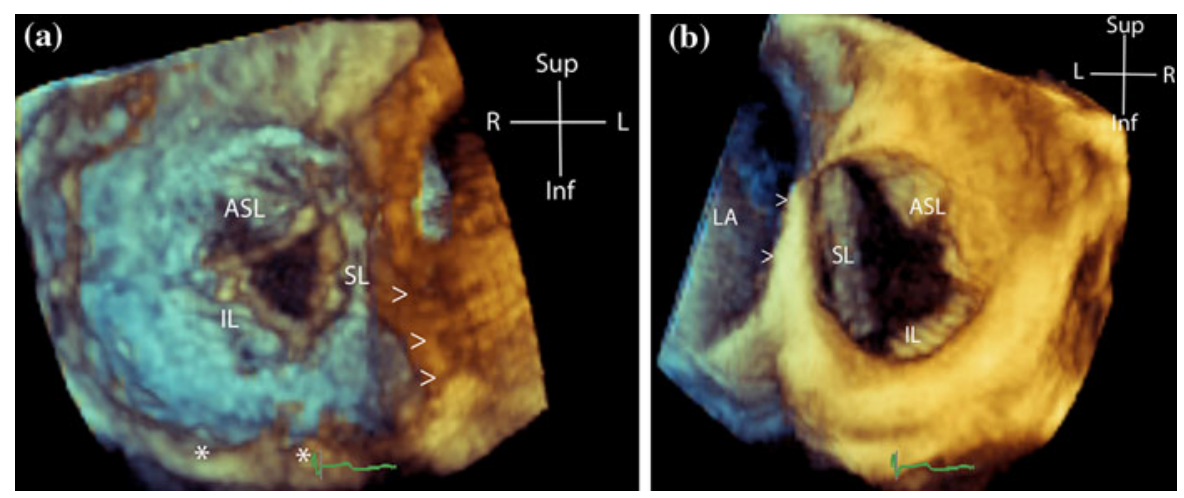

Fig. 5 a En face view of the tricuspid valve viewed from the right ventricle. The image is anatomically orientated so that the inferior margin of the right ventricle is lowermost in the image. The ventricular septum is marked by the arrows $(>)$ with the adjacent septal leaflet of the tricuspid valve. The diaphragmatic surface of the heart is marked by asterisks. ASL antero-superior leaflet of the tricuspid valve, $I L$ inferior leaflet of the tricuspid valve, SL septal leaflet of the tricuspid valve, asterisks diaphragmatic surface of the heart, arrows ventricular septum.

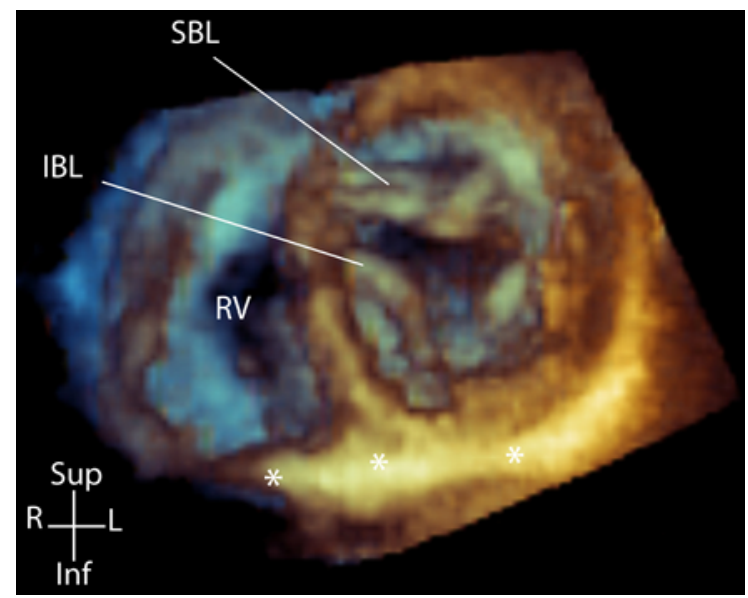

Fig. 6 Left atrioventricular valve in AVSD viewed from the left ventricle. The diaphragmatic surface is marked by the asterisks. The superior bridging leaflet is uppermost on the image and inferior bridging leaflet is lowermost making anatomic interpretation intuitive. $S B L$ superior bridging leaflet of left atrioventricular valve, $I B L$ inferior bridging leaflet of the left atrioventricular valve., $R V$ right ventricle, asterisks diaphragmatic surface of the heart, Sup superior, Inf inferior, $L$ left, $R$ right

prefer to maintain this display convention. However, using 3D echocardiography, the aortic valve may also be viewed as if looking through the left ventricular outflow tract from the left ventricle. In this instance the anterior structures such as the pulmonary artery can be displayed uppermost (Fig. 11).
Sup superior, Inf inferior, $R$ right, $L$ left. b View of the tricuspid valve from the right atrium. The three leaflets of the tricuspid valve can be visualised as if viewed from the right atrium looking into the right ventricle. The supero-inferior orientation is identical to Fig. 5a. The atrial septum is indicated with the arrows $(>)$ and the left atrium is to the left side of the image. $L A$ left atrium, $A S L$ antero-superior leaflet of the tricuspid valve, $I L$ inferior leaflet of the tricuspid valve, $S L$ septal leaflet of the tricuspid valve, Sup superior, Inf inferior, $R$ right, $L$ left

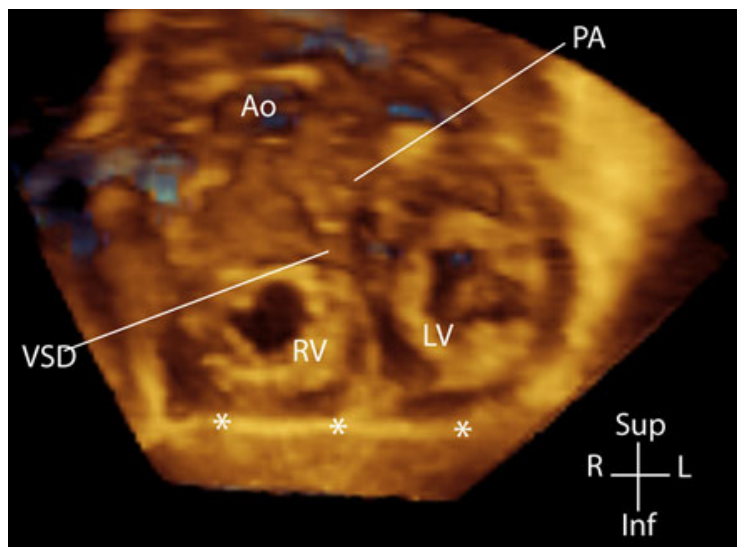

Fig. 7 Subcostal view of an infant with ventriculo-arterial discordance (Transposition of the Great Arteries), ventricular septal defect (VSD) and pulmonary valve stenosis. The diaphragmatic plane is marked $(*)$ and permits reference to the inferior border of the heart whatever the cropped projection. The relationship of the left ventricle and VSD to the aorta can be assessed. Ao aorta, $P A$ pulmonary artery, VSD ventricular septal defect, $L V$ left ventricle, $R V$ right ventricle, asterisks diaphragmatic surface of the heart, Sup superior, Inf inferior, $L$ left, $R$ right

\section{Pulmonary valve}

The pulmonary valve is often technically difficult to image due to its thin leaflets and anterior position just behind the sternum. Nonetheless 3D echocardiography can permit en face visualisation of the pulmonary 


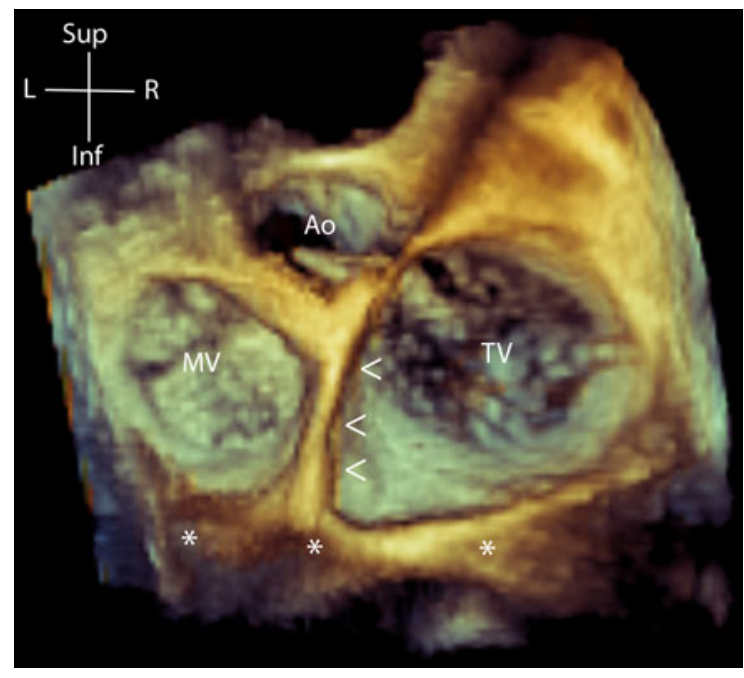

Fig. 8 "Surgical" view of the atrioventricular valves. Transoesophageal 3D echocardiographic view of the atrioventricular junction. The image is orientated in such a way that the mitral valve (MV) is to the left of the image and the tricuspid valve (TV) is to the right. The diaphragmatic surface of the heart (*) is lowermost and the aorta shown more superiorly on the image. The atrial septum is shown by the arrows (>). $M V$ mitral valve, $T V$ tricuspid valve, Ao aorta, asterisks diaphragmatic surface of the heart. Arrows atrial septum, Sup superior, Inf inferior, $R$ right, $L$ left

valve from the pulmonary artery side but perhaps more usefully from the right ventricular outflow tract (Fig. 12).

\section{The atrial septum}

Three-dimensional echocardiography is being employed extensively to complement conventional cross-sectional imaging to investigate atrial septal defects and to guide catheter intervention. One of the clinical aims is to optimise the selection of potential candidates for transcatheter device closure versus surgical repair of the atrial septal defect. Novel en face projections of atrial septal defects are used extensively to outline the shape, location and size of the defect $[10,11]$. Such views of the atrial septum can either be from a right atrial (Fig. 13) or from the left atrial aspect (Fig. 14) and are routinely processed from the same 3D dataset. Consistent anatomic orientation of en face views can be used during surgical or interventional planning and for "real time" echocardiographic guidance of a catheter intervention.

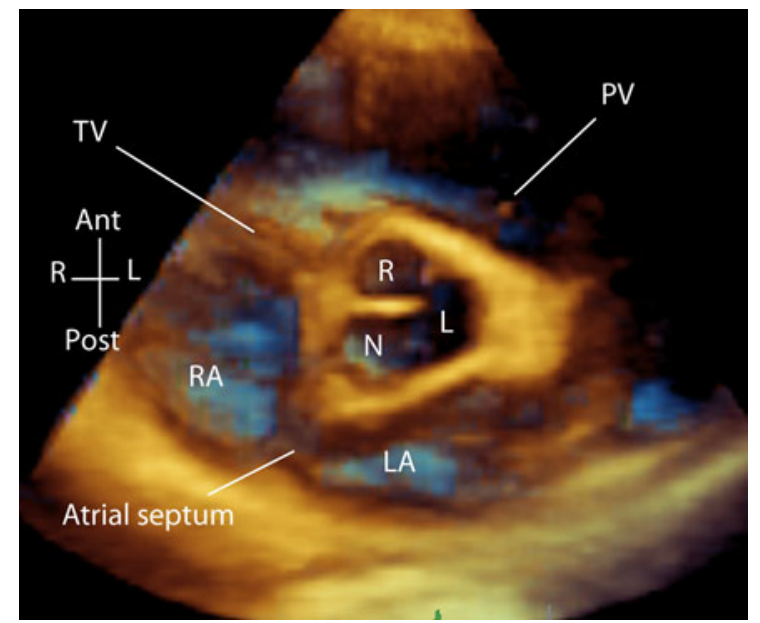

Fig. $93 \mathrm{D}$ transthoracic short axis view of the aortic valve, analogous to that of conventional $2 \mathrm{D}$ short axis view. The tricuspid valve is seen to the left of the image and the pulmonary valve to the right of the image. $T V$ tricuspid valve, $P V$ pulmonary valve, $R A$ right atrium, $L A$ left atrium; Aortic Valve Cusps ( $R$ right, $L$ left, N-non-coronary). Orientation tool: Ant anterior, Post posterior, $L$ left, $R$ right

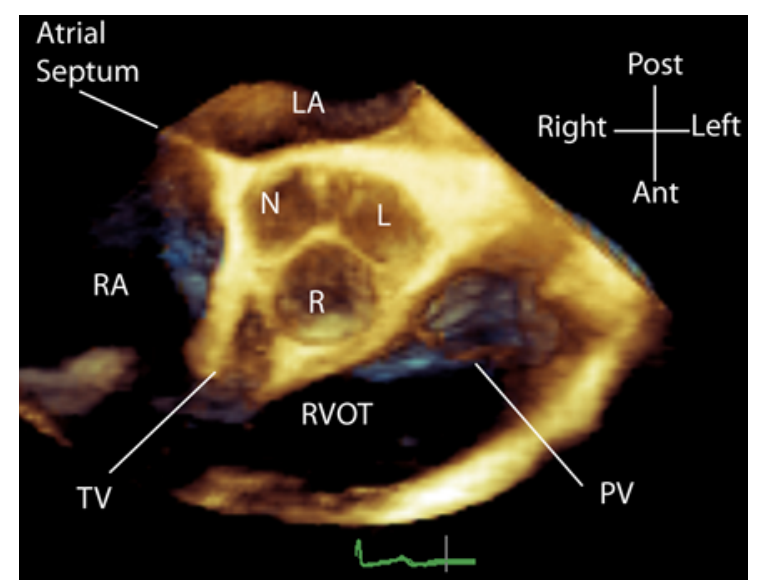

Fig. 10 Transoesophageal 3D echocardiographic view of the aortic valve using the conventional orientation. Although this view is not oriented anatomically as the more anterior-superior structures are shown lowermost on the image, this orientation may be more familiar to many echocardiographers. $T V$ tricuspid valve, $P V$ pulmonary valve, $R A$ right atrium, $L A$ left atrium, $R$ right coronary cusp, $N$ non-coronary cusp, $L$ left coronary cusp, Post posterior, Ant anterior

\section{The ventricular septum}

En face views of the ventricular septum can delineate the size, shape, location and anatomical relations of ventricular septal defects in preparation for surgical 


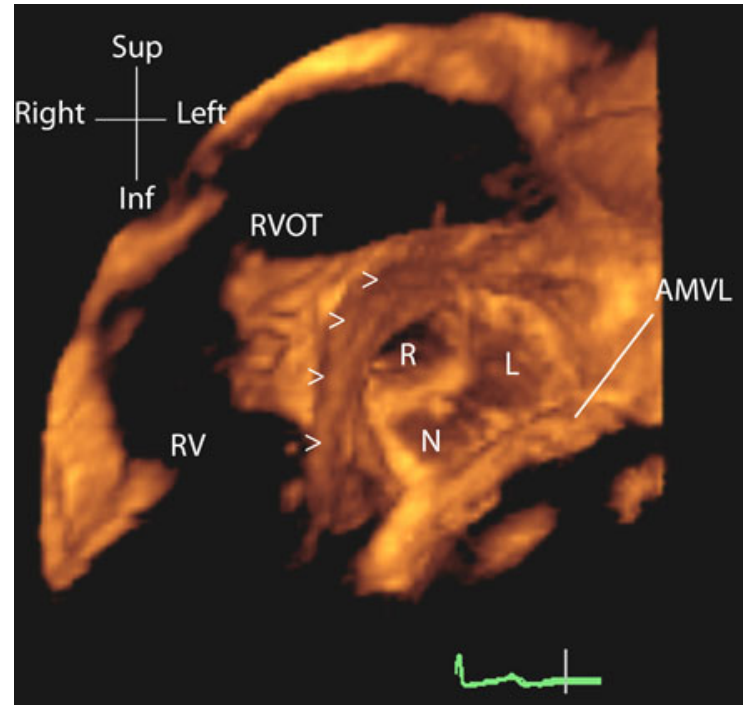

Fig. 11 3D echocardiographic view of the aortic valve viewed from the left ventricle. The imaging plane used is similar to that demonstrated in Fig. 4b, c but the cut plane is extended further towards the aortic valve so that only the anterior mitral valve leaflet (AMVL) is visible. The right coronary cusp (R) appears superior and leftwards on the image and the left coronary cusp (L) appears to the right on the image. The right ventricle (RV) and right ventricular outflow tract can be seen to wrap around the left ventricle. The plane of the ventricular septum is arrowed ( $>$ ). $R V$ right ventricle, $R V O T$ right ventricular outflow tract, $A M V L$ anterior mitral valve leaflet, $R$ right coronary cusp, $N$ non-coronary cusp, $L$ left coronary cusp, Sup superior, Inf inferior, arrows ventricular septum

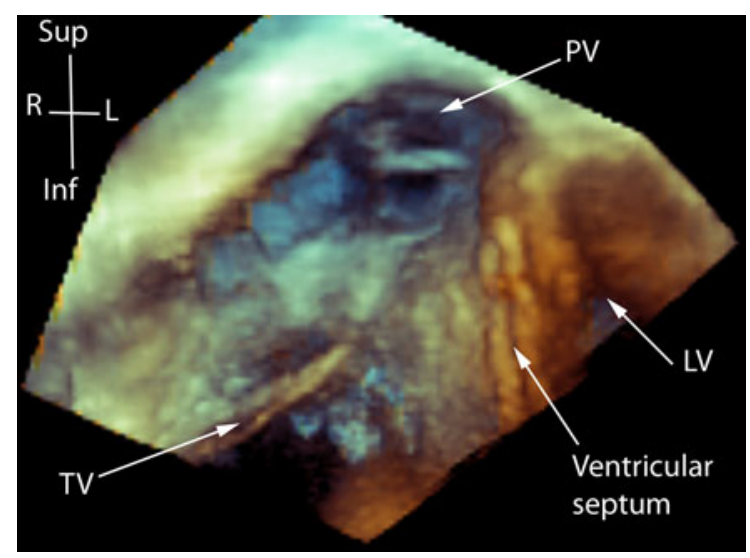

Fig. $123 \mathrm{D}$ projection of the right ventricular outflow tract leading to the pulmonary valve in the far field. $P V$ pulmonary valve, $T V$ tricuspid valve, $L V$ left ventricle, Sup superior, Inf inferior, $L$ left, $R$ right

repair or to assess for catheter closure. The 3D dataset can be cropped from either side to reveal an uninterrupted projection of the right or left ventricular septal

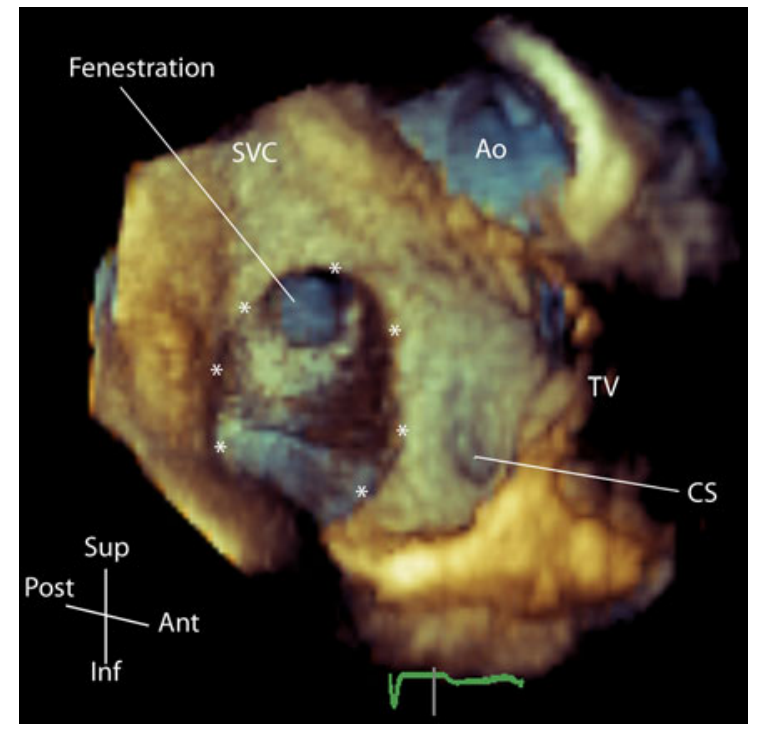

Fig. 13 3D transoesophageal echocardiographic view of a fenestrated atrial septal defect viewed from a right atrial projection. The image is orientated so that the superior vena cava (SVC) are uppermost in the image. The aorta (Ao) is seen antero-superiorly and the mouth of the coronary sinus (CS) is seen inferiorly. The tricuspid valve (TV) is to the right on the image. The margins of the oval fossa are highlighted with asterisks. SVC superior vena cava, Ao aorta, $T V$ tricuspid valve, CS coronary sinus, Sup superior, Inf inferior, Ant anterior, Post posterior

surface. As such cropping by necessity removes readily identifiable anatomic structures for example the moderator band in the right ventricle, the use of anatomical orientation displays superior structures uppermost and the apical portion of the right ventricle to the right when viewed from the RV (Fig. 15a) and the apical portion of the left ventricle shown to the left when viewed from the left ventricle (Fig. 16a). Figures $15 \mathrm{~b}$ and $16 \mathrm{~b}$ demonstrate comparable projections after device occlusion of a ventricular septal defect.

\section{Use of multiplanar reformatted techniques}

Appropriate orientation of 3D echocardiographic images is relatively easily achieved for rendered 3D views because most software permits rotation of the image in the z-plane. Some software packages will permit measurements of distance and area on the rendered views and others not. Thus, multiplanar reformatted images are often used to make distance and area measurements (Fig. 17a). Furthermore, the multiplanar technique permits systematic analysis of 


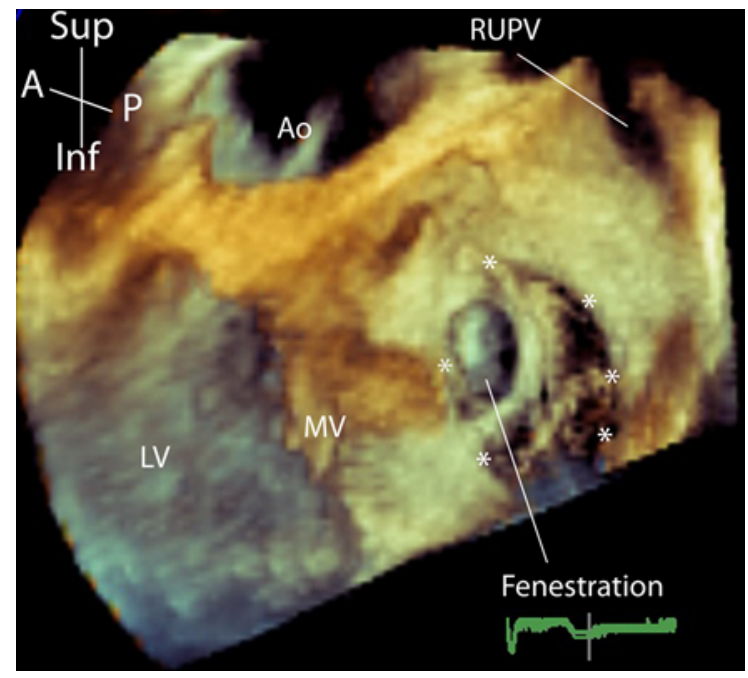

Fig. $143 \mathrm{D}$ transoesophageal echocardiographic image of a fenestrated atrial septal defect viewed from a left atrial projection. Superior structures are uppermost. The mitral valve and left ventricle are shown to the left of the image. The antero-superior relationship of the aorta (Ao) to the atrial septal defect is maintained and the supero-inferior orientation is consistent when compared to the right atrial projection (Fig. 13). Ao aorta, $R U P V$ right upper pulmonary vein, $M V$ mitral valve, $L V$ left ventricle, asterisks margins of the oval fossa, Sup superior, Inf inferior, $A$ anterior, $P$ posterior

the 3D volumetric datasets by user-defined planes for more complex congenital heart defects (Fig. 17b). Depending on the analysis package, it may be difficult or impossible to rotate such multiplanar images to an anatomic position. However, the availability of the other cross-referencing planes, and reference to rendered images assists the user in this regard (Fig. 17b).

\section{Discussion}

When investigating congenital heart disease, 3D echocardiography can provide new ways of displaying abnormal anatomy and is particularly useful for analysing complex spatial relations. Projections such as en face visualisation of the cardiac septums or the atrioventricular valves can provide hitherto unavailable echocardiographic views which may further enhance surgical or interventional management. In contrast to other 3D modalities (MRI and CT) there has been little discussion about image orientation of $3 \mathrm{D}$ echocardiographic data. In most congenital heart centres, decisions about patient management are made at multi-disciplinary meetings during which cardiologists, surgeons and other imaging specialists review data from multiple imaging modalities including echocardiography, MRI, catheter angiography and CT scanning. Presenting 3D echocardiographic images in anatomic format is consistent with other established imaging techniques, notably MRI and CT. In our experience, this approach has been well received particularly by surgeons and other nonechocardiographers who have not been primarily
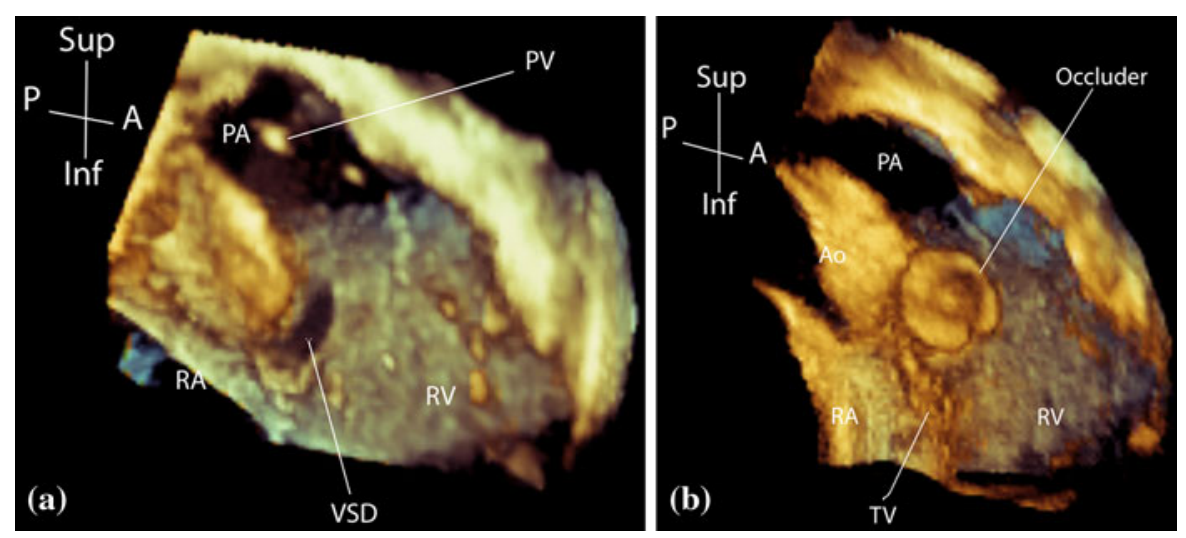

Fig. 15 a Three dimensional echocardiographic view of the ventricular septum viewed from the right ventricular aspect demonstrating a ventricular septal defect (VSD). $P A$ pulmonary artery, $P V$ pulmonary valve, $R V$ right ventricle, $R A$ right atrium, Sup superior, Inf inferior, $A$ anterior, $P$ posterior. b Three-dimensional echocardiographic view of the ventricular septum viewed from the right ventricular aspect following deployment of an occlusion device into the ventricular septal defect. $P A$ pulmonary artery, $T V$ tricuspid valve, $R V$ right ventricle, $R A$ right atrium, Sup superior, Inf inferior, $A$ anterior, $P$ posterior 

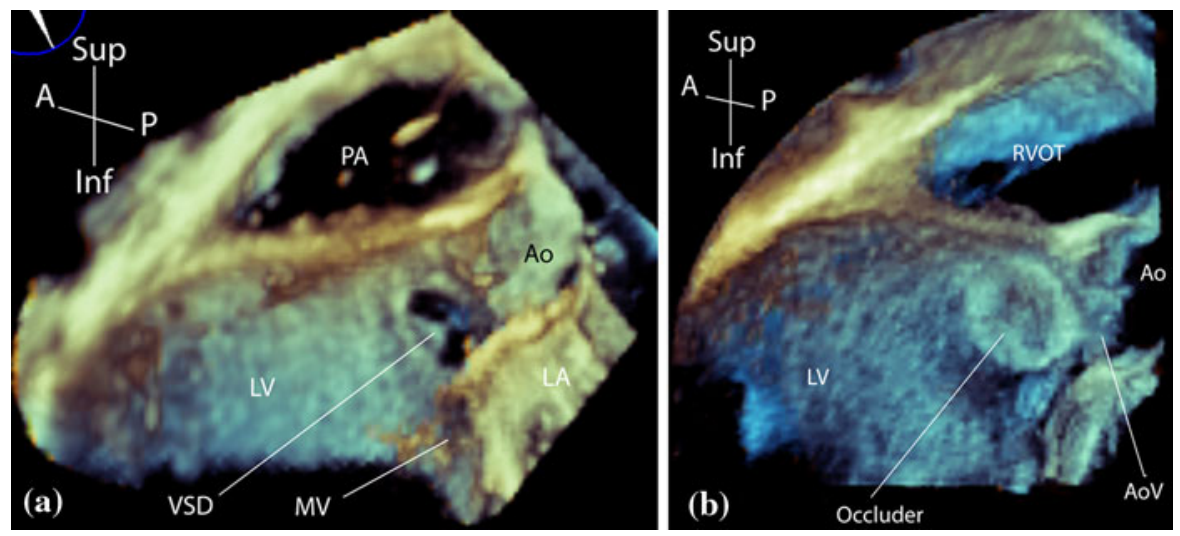

Fig. 16 a Three-dimensional echocardiographic view of a ventricular septal defect (VSD) from the left ventricular aspect. In this projection, the relationship of the aorta (Ao) to the ventricular septal defect including intervening tissue between the aortic valve and the defect. This image maintains the same supero-inferior orientation as Fig. 15a, b. The echocardiographic dataset has been rotated around a vertical axis to move from a projection from the right ventricular aspect to one from the left ventricular aspect. $P A$ pulmonary artery, $A o$ aorta, $L V$ left ventricle, $M V$ mitral valve, $V S D$ ventricular septal defect, $L A$ left atrium. Sup superior, Inf inferior, $A$ anterior, $P$ posterior. b Three-dimensional echocardiographic image of a ventricular septal defect occluder viewed from the left ventricular aspect. The relationship of the occluder to the aortic valve (AoV) can be clearly visualised. The projection is identical to that used in a to allow pre and post intervention comparison. RVOT right ventricular outflow tract, $A o$ aorta, $A o V$ aortic valve, $L V$ left ventricle, Sup superior, Inf inferior, $A$ anterior, $P$ posterior

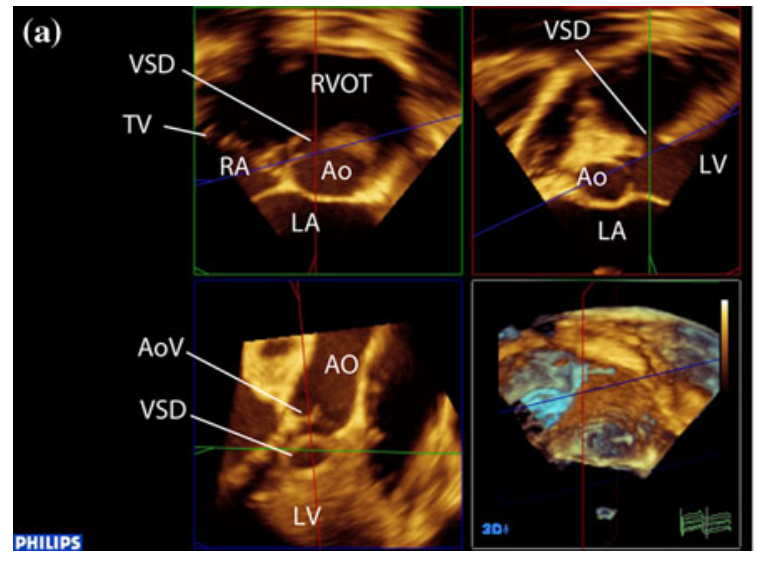

Fig. 17 a Multiplanar reformatted images of a perimembranous ventricular septal defect. This modality is frequently employed to measure defects such as ventricular septal defects. The blue orientation plane has been rotated so that the lower left pane shows the ventricular septal defect en face. This permits measurement of the length, diameter and area of the defect to be measured. $L A$ left atrium, $R A$ right atrium, $L V$ left ventricle, $V S D$ ventricular septal defect, $T V$ tricuspid valve, $A o$ aorta, AoV aortic valve, RVOT right ventricular outflow tract. b Multiplanar reformatted images of a heart with transposed

involved in image acquisition. This approach is particularly important for more complex forms of congenital heart disease where the usual anatomical relationships of one cardiac segment to another cannot be taken for granted.

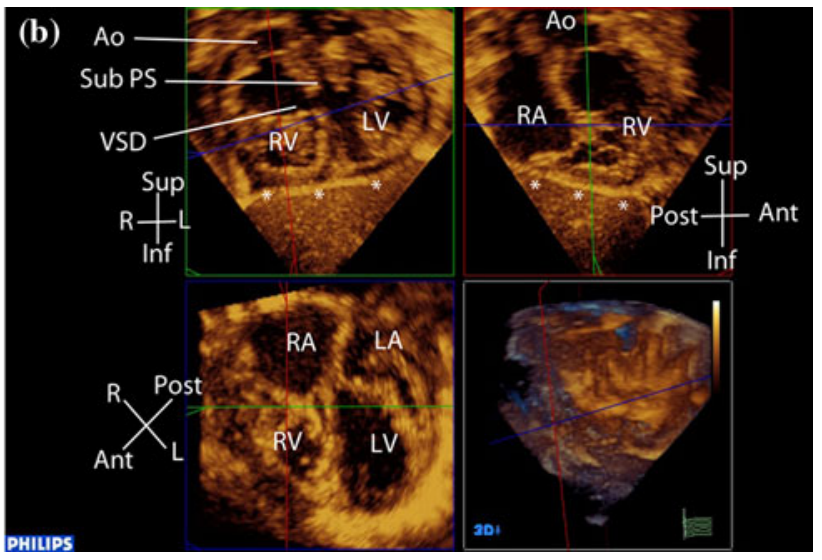

great arteries, ventricular septal defect and subpulmonary stenosis. The different quadrants show different planes through the heart. Each of the red, blue and green imaging planes can be moved or rotated to produce the desired plane of interrogation. The diaphragmatic surface is marked with asterisks which assists in image orientation. LA left atrium, $R A$ right atrium, $L V$ left ventricle, $R V$ right ventricle, Sub $P S$ subpulmonary stenosis, Ao aorta, $V S D$ ventricular septal defect, Sup superior, Inf inferior, $L$ left, $R$ right, Ant anterior, Post posterior, asterisks diaphragmatic surface

In conclusion, the adoption of an anatomic approach to the display of $3 \mathrm{D}$ echocardiographic information, consistent with other imaging modalities, has proved beneficial for the adoption of this technique into clinical decision-making. 
Acknowledgments The contribution of Anna Barlow, Kelly Nugent and Debbie Rawlins in the acquisition of some 3D echocardiograms is acknowledged. We acknowledge the financial support of the Evelina Children's Hospital Appeal in the purchase of echocardiography equipment.

\section{Conflict of interest None.}

\section{References}

1. Simpson JM (2011) Congenital heart disease in children. In: Buck T, Franke A, Monaghan M (eds) Three-dimensional echocardiography. Springer, Berlin, pp 201-219

2. Meijboom FJ, van der Zwaan H, McGhie J (2011) Threedimensional echocardiography in adult congenital heart disease. In: Buck T, Franke A, Monaghan M (eds) Threedimensional echocardiography. Springer, Berlin, pp 175-198

3. Simpson JM, Miller O (2011) Three-dimensional echocardiography in congenital heart disease. Arch Cardiovasc Dis 104(1):45-56
4. Hung J et al (2007) 3D echocardiography: a review of the current status and future directions. J Am Soc Echocardiogr 20(3):213-233

5. Lai WW et al (2006) Guidelines and standards for performance of a pediatric echocardiogram: a report from the Task Force of the Pediatric Council of the American Society of Echocardiography. J Am Soc Echocardiogr 19(12):1413-1430

6. Nanda NC et al (2004) Examination protocol for three-dimensional echocardiography. Echocardiography 21(8):763-768

7. Buck T (2011) Basic principles and practical application. In: Buck T, Franke A, Monaghan M (eds) Three-dimensional echocardiography. Springer, Berlin, pp 21-53

8. Anderson RH, Razavi R, Taylor AM (2004) Cardiac anatomy revisited. J Anat 205(3):159-177

9. Lang RM et al (2006) Recommendations for chamber quantification. Eur J Echocardiogr 7(2):79-108

10. Vaidyanathan B, Simpson JM, Kumar RK (2009) Transesophageal echocardiography for device closure of atrial septal defects: case selection, planning, and procedural guidance. JACC Cardiovasc Imaging 2(10):1238-1242

11. Mathewson JW et al (2004) Absent posteroinferior and anterosuperior atrial septal defect rims: Factors affecting nonsurgical closure of large secundum defects using the Amplatzer occluder. J Am Soc Echocardiogr 17(1):62-69 